\title{
Toward Optimal Cross-layer Solutions for Cognitive Radio Wireless Networks
}

\author{
Francesco Lo Presti \\ Dipartimento DISP \\ Università di Roma "Tor Vergata" \\ Email: lopresti@info.uniroma2.it
}

\author{
Chiara Petrioli \\ Dipartimento di Informatica \\ Università di Roma "La Sapienza" \\ Email: petrioli@di.uniroma1.it
}

\begin{abstract}
Cognitive radio (CR) networks have been proposed as a viable solution to spectrum scarcity problems. In CR networks, CR nodes exploit spectrum holes in space, time and/or frequency to transmit on licensed frequency bands without affecting primary users. In such a dynamic and unpredictable environment, CR networks require the ability to gather information on the surrounding available spectrum and to exploit this information to maximize CR nodes performance. In a companion paper we deal with sensing architecture and protocols. In this paper, instead, we derive a cross-layer scheme for cognitive radio networks which jointly optimize the sources flow rates, routing and medium access control while accounting for and exploiting the available spectrum resources. The proposed scheme builds on important recent results on close to optimal fully distributed CSMA-based scheduling algorithms, which allows us to derive a fully distributed solution.
\end{abstract}

\section{INTRODUCTION}

Cognitive Radio networks (CR) have recently emerged as promising solutions to improve spectrum efficiency. In a cognitive radio network so called secondary nodes opportunistically exploit spectrum holes in space, time and/or frequency to transmit on licensed frequency bands while ensuring that the performance of licensed users (also called primary users) are not detrimentally affected.

Differently from traditional wireless networks, CR networks require some form of spectrum sensing, i.e., the ability to detect primary user activities over possibly a wide range of frequencies, along with mechanisms for dynamic spectrum allocation. Spectrum sensing has the objective to identify which part of the spectrum can be used by secondary users, where and when, so that the level of interference generated by secondary users signals, measured at the location of primary receivers, is under a tolerable level. Most approaches so far proposed put the burden of spectrum sensing on the secondary user themselves. In these scenarios, the secondary nodes proactively sense the spectrum to locate available spectrum holes to be used for transmissions. Recently there have been some proposals which envision the existence of a specialized sensor network, designed, deployed and optimized for spectrum monitoring [4], [15]. In such scenarios, the secondary users interact with the sensor network to learn about spectrum availability and negotiate bandwidth usage. The advantages

This work is supported by the European Communitys Seventh Framework Programme (FP7/2007-2013) under grant agreement n 216076 (SENDORA). of this solution is that secondary nodes need no specialized hardware: hardware/software complexity involved with the spectrum monitoring is kept within the sensor network itself.

Once schemes for cooperative spectrum sensing have been selected, cognitive radio networks spectrum access techniques must be designed to take advantage of the spectrum holes as they occur. Many spectrum access solutions have been proposed (see [8] and reference therein for a recent survey). They differ in terms of reference architecture (infrastructures based vs ad-hoc), decision strategy (centralized vs decentralized), presence of a common control channel, access strategy (random access vs time slotted).

In this paper, we study the problem of dynamic spectrum allocation from a different direction as we attempt to identify a completely distributed cross-layer solution for Cognitive Radio networks. Our contribution is twofold. We start with the formulation of a specialized version of the well known network utility based maximization (NUM) problem [7], [6], [11], [9] (to name a few), which accounts for the limited availability of spectrum resources due to primary users activity. As first contribution, by means of standard duality theory, we derive an ideal optimal cross-layer decentralized scheme for rate control, routing, scheduling and spectrum access control that can be regarded as a distributed solution of the given optimization problem. In the proposed solution, secondary nodes route traffic and schedule transmission according to a back-pressure scheduler [17], which uses the differential queue length at two end nodes of a link to determine links schedule. Spectrum access is carried out according to a simple fully distributed CSMA algorithm with exponential backoff, which is function of both queue length and spectrum availability. The latter key result can be derived from the distributed adaptive CSMA algorithm recently presented in [10], which has been shown to achieve throughput optimality in a distributed way. We remark that this is an ideal protocol analytically derived as an iterative solution of the dual of the proposed optimization problem. As such it cannot be directly implemented.

As second contribution we discuss how to derive an actual implementation from the proposed ideal scheme. In particular, we identify the requirements in terms of which type of information regarding spectrum availability should be made available to Cognitive Radio users and when to perform optimal spectrum access. All the required functionalities can be actu- 
ally implemented by means of existing architectures/schemes. We suggest the use of a dedicated wireless sensor network [4], [15] to carry out the sensing tasks. Cognitive Radio nodes query the sensor network to gather spectrum information availability. With this information available, Cognitive nodes compete for the available channels using a CSMA link backoff scheme. A common control channel is used to exchange RTS/CTS message which besides implementing the necessary collision avoidance mechanism allows the sender to communicate the actual transmission frequency to the receiver.

Recently, different cross-layer solutions which explore the interactions among layers have been proposed for cognitive radio networks [5], [2], [3]. The different approaches differ substantially in terms of assumptions and objective to be directly comparable with the proposed solution. In particular, differently from prior works our solution takes advantage of the recent result [10] which allows us to identify an optimal, albeit ideal, spectrum access strategy for cognitive radio users.

The rest of the paper is organized as follows. In Section II we describe the cognitive radio model and formulate network utility optimization problem. In Section III we present the rate control, scheduling and spectrum access protocols we as derived from the model. In Section IV we formally derive the different algorithms. Section V concludes the paper.

\section{Model AND Problem Formulation}

\section{A. Secondary Network Model}

We model the secondary network with a directed multigraph $G=(N, L)$ where nodes represent secondary nodes and edge $l$ the communication link, or sublink, between node $n$ and $m$ at frequency $c$. We will refer to $n=h(l), m=t(l)$ and $c(l)$ as the head, tail and channel of the sublink, respectively. The set of sublinks $l \in L$ with the same head $h(l)=n$ and tail $t(l)$ form a logical link $(n, m)$ between nodes $n$ and $m$. We assume that $c$ takes values in a finite set $C=\left\{c_{1}, \ldots, c_{k}\right\}$ of available frequencies. For the sake of simplicity, we assume that the set $C$ does not depend on the nodes/links. Communication links between node $n$ and $m$ exist if and only if node $n$ and $m$ are within transmission range of each other. For each node $n$, we denote by $L o(n):=\{l \in L \mid h(l)=n\}$ and $L i(n):=\{l \in L \mid t(l)=n\}$, the set of sublinks originating and terminating at node $n$, respectively. For symmetry, we assume that if $(n, m ; c) \in L$ then $(m, n ; c) \in L$ as well. Each sublink $l \in L$ is characterized by a constant $\alpha_{l}$, the sublink availability, which represents the fraction of time sublink $l$ is not occupied by primary transmission and is thus available for secondary transmissions.

\section{B. Traffic Model}

We model network traffic by a set of end-to-end flows $F$. Each flow $f \in F$ is characterized by a source node $s(f)$, a destination node $d(f)$ and the flow rate $x_{f}$. Flow traffic is routed by the network along one or multiple paths from source to destination. We denote by $s_{f l}$ the rate of flow $f$ on link $l$.
The flow conservation law implies that for $f \in F, n \in N$ :

$$
\begin{aligned}
x_{f}+\sum_{l \in L i(n)} s_{f l} & =\sum_{l \in L o(n)} s_{f l} \quad n=s(f), f \in F \\
\sum_{l \in L i(n)} s_{f l} & =\sum_{l \in L o(n)} s_{f l} \quad n \neq s(f), d(f), f \in F
\end{aligned}
$$

which simply states that for any node traversed by the flow $f$, except for the destination node $d(f)$, the flow entering the node must be equal to the flow exiting it.

We associate a utility function $U_{f}\left(x_{f}\right)$ with each flow $f \in F$, which is assumed to be differentiable, strictly concave, non decreasing. There are several class of functions satisfying this condition, e.g., $\log \left(1+x_{f}\right)$ and $\beta_{f} \frac{x_{f}^{1-\alpha_{f}}}{1-\alpha^{f}}$ which are used to characterize a large class of fairness concept including weighted proportional and max-min fairness [13]. Finally, we assume utility is additive so that the aggregate traffic utility is $U(x)=\sum_{f \in F} U_{f}\left(x_{f}\right)$.

\section{Interference/Conflicts Model}

We use a conflict graph to capture scheduling constraints among different sublinks. A conflitc graph is a graph $\mathcal{G}=$ $(\mathcal{V}, \mathcal{E})$, where $\mathcal{V}$ is the set of vertices, each of them representing a sublink, and $\mathcal{E}$ is the set of edges, representing sublinks conflicts, i.e., two links cannot transmit at the same time if and only if there is an edge between them.

We need to consider different types of conflicts. First, because of the shared wireless channels, transmissions over different sublinks causes interference when the sender of one sublink is within the interference range of the sender or the receiver of another sublink and the two sublinks share the same channel. Second, we need to account for the physical limitation that one node can transmit over only one single channel at a given time. Thus, for any node $n \in N$, sublink $l$ also conflicts with all links $l^{\prime} \in L o(n), l^{\prime} \neq l, c(l) \neq c\left(l^{\prime}\right)$.

Conflict graphs allow us to identify the sets of sublinks which can transmit simultaneously.

Definition An independent set of the conflict graph $\mathcal{G}=$ $(\mathcal{V}, \mathcal{E})$ is a subset $I \in \mathcal{V}$ such that no two elements are adjacent, i.e., $i, j \in I \Rightarrow(i, j) \notin \mathcal{E}$. Only sublinks within the same independent set can transmit simultaneously without interference/conflict with each other.

Let $\mathcal{I}$ denote the set of independent sets of $\mathcal{G}$. We denote by $a_{I}=\left(a_{I l}\right)_{l \in L}, I \in \mathcal{I}$, a binary vector that indicates which link belong to $I: a_{I l}=1$ if $l \in i$ and $a_{I l}=0$ otherwise. We will denote by $\mathcal{X}=\left\{a_{I} \mid I \in \mathcal{I}\right\}$ the set of all characteristics vectors $a_{I}$.

MAC protocols differ in how nodes contend and are granted access to the available channels. Since only independent sets can be simultaneously scheduled, over the long run different protocols can be characterized by the frequency $p_{I}$ according to which transmission over the independent set $I \in \mathcal{I}$ occur, $\sum_{I \in \mathcal{I}} p_{I}=1$. From these frequencies, we directly obtain an expression for the average transmission rate over sublink $l$ as $\alpha_{l} \sum_{I \in \mathcal{I}} p_{I} a_{l}^{I}$ under the assumption of unit capacity. 


\section{Problem Formulation}

An attractive solution for the secondary network consists in designing network protocols capable of exploiting the dynamically available spectrum holes as to maximize the aggregate traffic utility.

We derive these protocols from the solution of the utility maximization problem which takes the following form subject to resource constraints:

$$
\begin{aligned}
\text { P: } \max & \sum_{f \in F} U_{f}\left(x_{f}\right) \\
\text { s.t. } & x_{f}+\sum_{l \in L i(n)} s_{f l} \leq \sum_{l \in L o(n)} s_{f l} \quad n=s(f), f \in F \\
& \sum_{l \in L i(n)} s_{f l} \leq \sum_{l \in L o(n)} s_{f l} \quad n \neq s(f), d(f), f \in F \\
& \sum_{f \in F} s_{f l} \leq \alpha_{l} \sum_{I \in \mathcal{I}} p_{I} a_{l}^{I} \quad l \in L \\
& \sum_{I \in \mathcal{I}} p_{I}=1, \quad p_{I} \geq 0 \quad I \in \mathcal{I}
\end{aligned}
$$

Constraints (3)-(4) are the flow constraints which we find convenient to express as inequalities. Constraints (5)-(6) simply state that the average flow rate traversing a sublink cannot exceed the average transmission rate of that sublink.

\section{Joint RATE, SChEDUling AND MAC CONTROL FOR SECONDARY NODES}

The solution of the network utility maximization problem $\mathbf{P}$ via Lagrangian duality implicitly defines an ideal distributed cross-layer scheme for joint rate control, scheduling and MAC of the secondary network. In this section we present the protocols/algorithms themselves. We formally derive them in Section IV.

Backlogged secondary nodes access the network according to a CSMA like protocol with an exponential backoff timer. Each node $n$ maintains a separate queue for each flow $f$ traversing it. We let $Q_{n f}$ denote this queue length. Also associated to each flow, the node keeps a control variable $q_{n f}$, which is proportional to the queue length $Q_{n f}$ (and that we will see represent a Lagrangian variable of the Dual problem). Source nodes also keep track of the flow rate $x_{f}$.

We assume nodes update the values of the variables $q_{n f}$, and $x_{f}$ periodically at time $t_{j}=j T$, where $T$ is a design parameter. The values are kept constant for the entire next period up to $t_{j+1}=(j+1) * T$. Initially all nodes queues are empty and $q_{n f}=0$. We now describe the protocols/algorithm in details.

\section{A. Scheduling}

Nodes use a queue-length based scheduler known as the back-pressure scheduler [17], which uses the differential queue length at the two end nodes of a link to determine the link schedule. This scheduling algorithm which can be shown to achieve throughput optimality [17] works as follows. At the beginning of each period $t_{j}$ node $n \in N$ computes for each outgoing link $(n, m)$ the maximal back-pressure between its queues and its downlink node queues $z_{n m}=$ $\max _{f \in F} q_{n f}-q_{m f}$. Let $f^{*}=\operatorname{argmax}_{f \in F} q_{n f}-q_{m f}$ be the flow with maximal back-pressure along link $(n, m)$. During interval $\left(t_{j}, t_{j+1}\right]$ node $n$ schedules for transmission on all sublinks $l=(n, m ; c) \in L o(n), c \in C$, flow $f^{*}$ traffic (only).

\section{B. MAC protocol for secondary nodes}

The MAC protocol employed by the secondary nodes is inspired by recent results in [10].

Whenever node $n \in N$ has backlogged traffic to send over one of its outgoing link $(n, m)$ (as determined by the scheduling), it waits for an exponentially distributed amount of time with rate $R_{n m}=\sum_{l \in(n, m)} R_{l}$ where $R_{l}=\frac{\exp \left(\alpha_{l} z_{n m}\right)}{\alpha_{l}}$. When the timer expires, the channel is determined randomly: channel $c$ is chosen with probability $p_{c}=\frac{R_{l}}{R_{n m}}, l=(n, m ; c)$. The channel is then sensed. If the channel is free the node transmits a flow $f^{*}$ packet; we assume transmission time is exponentially distributed with mean 1 . If the channel is busy, the node simply restarts the timer for that link. Observe that the channel is determined before sensing. A secondary node can find the channel busy either because a conflicting node started transmission, or because a primary node is using the same frequency. Sublink $l$ is found occupied by a primary node transmission with probability $1-\alpha_{l}$ and free with probability $\alpha_{l}$.

\section{Rate Control}

Source nodes updates the rate $x_{f}$ by computing the maximizer of $\beta U_{f}\left(x_{f}\right)-q_{s(f) f} x_{f}$. It is easy to realize that the maximizer is simply $x_{f}^{*}=U_{f}^{\prime-1}\left(q_{s(f) f} / \beta\right)$.

\section{Dual Variables Update}

Backlogged nodes updates the control variables $q_{n f}$ as follows (which will turn out to be the subgradient algorithm).

$$
\begin{aligned}
& q_{n f} \\
& =\left[q_{n f}-\gamma\left(x_{f}(\boldsymbol{q})+\sum_{l \in L i(s(f))} s_{f l}(\boldsymbol{q})-\sum_{l \in L o(s(f))} s_{f l}(\boldsymbol{q})\right)\right]^{+} \\
& =\left[q_{n f}-\gamma\left(\sum_{l \in L i(n)} s_{f l}(\boldsymbol{q})-\sum_{l \in L o(n)} s_{f l}(\boldsymbol{q})\right)\right]^{+} \\
& n \neq s(f), f \in F
\end{aligned}
$$

where $\gamma$ is a design parameter. Observe that $q_{n f} \propto Q_{n f}$ (it would be equal should $\gamma=T$, but typically $\gamma \ll 1$ as it ensures smoother behaviour of the queue length dynamics).

\section{E. Steps toward a Cross-Layer Solution}

The cross-layer solution just described is an ideal one and, as shown in Section IV, it can be formally derived as a distributed solution of the dual problem associated with $\boldsymbol{P}$. Clearly it cannot be directly implemented as presented. Now we discuss the main issues that need to be addressed. 
First, we need to define how to carry out spectrum sensing. In the proposed solution, we need that the secondary nodes know/measure/estimate $\alpha_{l}$, the fraction of time a given sublink is available for transmission. The availability of link $\alpha_{l}$, $l=(n, m ; c)$, is the fraction of time that a secondary user transmission over $l$ would not affect a primary transmission since it cannot cause interference at a primary receiver. As recently proposed, a viable solution is to let the secondary network units interact with a wireless sensor network that performs the spectrum sensing task and reports to secondary transmitters, through dedicated signalling channels, the required information periodically. We refer to a companion paper [14] for the description of such an architecture which can serve these objectives.

Second, we need to specify how the sender advertises the transmission frequency to the receiver and how the collision avoidance mechanism is implemented. We assume the secondary users share a global control channel[12], [16], [3]. At timer expiration, if the control channel is idle, backlogged nodes send an RTS message with the frequency information. On receiving the RTS, the receiver responds with a CTS message. These messages are also used to exchange queue length as required by the backpressure scheduler (as also suggested in [3]).

\section{Network Optimization Problem Solution}

In this section we show how the proposed cross-layer solution can be regarded as a distributed solution via Lagrangian duality to the NUM problem presented in Section II. We first establish some important results regarding the CSMA based MAC protocol.

\section{A. Stationary Distribution of the CSMA based MAC protocol}

Given the description of the MAC protocol in the previous section, it is not difficult to realize that the evolution of the secondary network can be modelled as a continuous Markov Chain $s(t)$ which takes values in $\mathcal{X} . s(t)=a_{I}$ if and only if all the sublinks in the independent set $I$ are transmitting. If the network is in state $a_{I}$ we have two types of transitions out of state $a_{I}$. If a link $l$ is not active $\left(a_{I l}=0\right)$ and all links conflicting with $l$ are not active as well, we have a transition from state $a_{I}$ to state $a_{I}+\boldsymbol{e}_{l}$ with rate $\alpha_{l} R_{l}$, where $\boldsymbol{e}_{l}$ is the $|L|$ dimensional vector with all element equal to 0 but the $l$-th which is equal to 1 . This transition represents link $l=(n, m)$ timer expiring, node $n$ choosing channel $c$ for transmission and sensing channel $c$. With probability $\alpha_{l}$ the channel is busy because a primary user is transmitting, the node restarts the timer. With probability $1-\alpha_{l}$ the channel is free and the node start transmitting over sublink $l$. If a sublink $l^{\prime}$ is active in state $a_{I}$, i.e. $a_{I l^{\prime}}=1$ we have a transition from state $a_{I}$ to state $a_{I}-e_{l^{\prime}}$ with rate 1 . No other type of transition are possible out of state $a_{I}$.

From $[10]^{1}$ it directly follows that the Markov chain $s(t)$

\footnotetext{
${ }^{1}$ The protocol discussed in [10] is actually different from the one presented here. Nevertheless, the modification we have introduced result into the exact same Markov Chain
}

has the following stationary distribution $\mathrm{P}\left[s(t)=a_{I} ; \boldsymbol{R}\right]$ :

$$
\mathrm{P}\left[s(t)=a_{I} ; \boldsymbol{R}\right]=\frac{\exp \left(\sum_{l \in L} a_{I l} \log \alpha_{l} R_{l}\right)}{C(\boldsymbol{R})}
$$

where $C(\boldsymbol{R})=\sum_{I \in \mathcal{I}} \exp \left(\sum_{l \in L} a_{I l}\left(\log r_{l}+\log \alpha_{l}\right)\right)$ and $\boldsymbol{R}=\left(R_{l}\right)_{l \in L}$. Hereafter, for the sake of simplicity, we shall write $p_{I}(\boldsymbol{R})$ in place of $\mathrm{P}\left[s(t)=a_{I} ; \boldsymbol{R}\right]$.

Moreover the stationary distribution of the CSMA Markov Chain $p(R)$ is the maximizer of the following expression

$$
-\sum_{I \in \mathcal{I}} p_{I} \log \left(p_{I}\right)+\sum_{I \in \mathcal{I}} p_{I} \sum_{l \in L i(n)} a_{I l} \log \alpha_{l} R_{l}
$$

over all possible distributions $\boldsymbol{p}=\left(p_{I}\right)_{I \in \mathcal{I}}$. This property will play an important role in deriving the distributed cross-layer solution for the secondary network.

\section{B. Solution of Network Optimization Problem $\mathbf{P}$}

Problem $\mathbf{P}$ is a convex optimization problem. Its direct solution would require a central entity with complete knowledge from the network topology to the flow utility function and would not be of practical interest. Here, we take advantage of the problem structure and derive a distributed solution via dual decomposition. Following [10] we actually consider a slightly different problem $\mathbf{P}^{\prime}$ obtained from $\mathbf{P}$ by replacing the objective function with

$$
-\sum_{I \in \mathcal{I}} p_{I} \log p_{I}+\beta \sum_{f \in \mathcal{F}} U_{f}\left(x_{f}\right)
$$

where $\beta>0$ is a weighting factor. We observe that the objective function of $\mathbf{P}^{\prime}$ is not the total utility because of the extra term $-\sum_{I \in \mathcal{I}} p_{I} \log p_{I}$. Nevertheless, as $\beta \rightarrow \infty$ the utility $\sum_{f \in \mathcal{F}} U_{f}\left(x_{f}\right)$ dominates the objective function so that the solution of $\mathbf{P}^{\prime}$ converges to the solution of $\mathbf{P}$. As we shall see later, this additional terms is required to allow an efficient distributed solution to the MAC layer sub-problem.

For the solution, we consider the following partial dual problem $\mathbf{D}^{\prime}$ associated with $\mathbf{P}^{\prime}$

$$
\begin{aligned}
\mathbf{D}^{\prime}: \min & D(\boldsymbol{q}) \\
\text { s.t. } & \boldsymbol{q} \geq 0
\end{aligned}
$$

with partial dual function

$$
\begin{array}{rlrl}
D(\boldsymbol{q})= & \max _{\boldsymbol{p}, \boldsymbol{s}, \boldsymbol{x}} L(\boldsymbol{p}, \boldsymbol{s}, \boldsymbol{x}, \boldsymbol{q}) & \\
\text { s.t. } & \sum_{f \in F} s_{f l} \leq \alpha_{l} \sum_{I \in \mathcal{I}} p_{I} a_{I l} & & l \in L \\
& \sum_{I \in \mathcal{I}} p_{I}=1, \quad p_{I} \geq 0 & & I \in \mathcal{I}
\end{array}
$$

and partial Lagrangian

$$
\begin{aligned}
L(\boldsymbol{p}, \boldsymbol{s}, \boldsymbol{x}, \boldsymbol{q})= & \sum_{f \in F}\left(U_{f}\left(x_{f}\right)-q_{s(f) f}\right)+\sum_{l \in L i(n)} s_{l}^{f}\left(q_{n f}-q_{m f}\right)+ \\
& -\sum_{I \in \mathcal{I}} p_{I} \log \left(p_{I}\right)
\end{aligned}
$$


where $\boldsymbol{q}=\left(q_{n f}\right)_{n \in N, f \in F}$ is the vector of Lagrangian multipliers associated to the flow constraints (3)-(4).

The dual problem $\mathbf{D}^{\prime}$ can be solved iteratively. At each step we update the value of the dual function $D(\boldsymbol{q})$ and compute a new iterate of the Lagrangian multipliers $\boldsymbol{q}$ as follows:

1) Dual Function Evaluation: Computation of $D(\boldsymbol{q})$ entails finding $\boldsymbol{p}$ (the MAC layer probabilities), $s$ (the routing and scheduling) and $\boldsymbol{x}$ (the rate control) which maximizes the Lagrangian (13). We first fix $\boldsymbol{p}$ and $\boldsymbol{x}$ and solve (12) for $\boldsymbol{s}$. We can compute the maximizer $s^{*}(\boldsymbol{q})$ by solving the following sub-problem:

$$
\begin{array}{cr}
\max _{s} \sum_{l \in L i(n)} s_{l}^{f}\left(q_{n f}-q_{m f}\right) & \\
\text { s.t. } \sum_{f \in F} s_{f l} \leq \alpha_{l} \sum_{I \in \mathcal{I}} p_{I} a_{l}^{I} & l \in L \\
s_{f l} \geq 0 & l \in L, f \in F
\end{array}
$$

The problem consists in maximizing a weighted sum of the rates $s_{f l}$ with weight $\left(q_{n f}-q_{m f}\right)$ with the constraints that sum of per flow rates $s_{f l}$ over a given link $l=(n, m ; c)$ is bounded by the average rate of that link. The solution is thus straightforward. For each link, let $z_{n m}=\max _{f \in F} q_{n f}-q_{m f}$. If $z_{n m}>0$ then for flow $f^{*}(\boldsymbol{q})=\operatorname{argmax}_{f \in F} q_{n f}-q_{m f}$ let $s_{l}^{f}=\alpha_{l} \sum_{I \in \mathcal{I}} p_{I} a_{l}^{I}$ and $s_{l}^{f}=0$ otherwise. If $z_{n m} \leq 0$, then set $s_{f l}=0$. This step corresponds to the back-pressure scheduling implemented by the nodes.

Plugging $\boldsymbol{s}(\boldsymbol{q})$ into (13), yields

$$
\begin{aligned}
D(\boldsymbol{q})= & \max _{\boldsymbol{p}, \boldsymbol{x}} L(\boldsymbol{p}, \boldsymbol{x}, \boldsymbol{q})= \\
& \max _{\boldsymbol{p}, \boldsymbol{x}} \sum_{f \in F}\left(\beta U_{f}\left(x_{f}\right)-q_{s(f) f} x_{f}\right)+ \\
& -\sum_{I \in \mathcal{I}} p_{I} \log \left(p_{I}\right)+\sum_{I \in \mathcal{I}} p_{I} \sum_{l \in L i(n)} a_{I l} \alpha_{l} z_{n m} \\
\text { s.t. } & \sum_{I \in \mathcal{I}} p_{I}=1, \quad p_{I} \geq 0 \quad I \in \mathcal{I}
\end{aligned}
$$

Since the Lagrangian is now separable in $\boldsymbol{p}$ and $\boldsymbol{x}$, computation of $x_{f}^{*}(\boldsymbol{q})$ and $p_{I}(\boldsymbol{q})$ can be carried out independently from each other. Under the assumption that $U_{f}$ is differentially invertible, $x_{f}^{*}(\boldsymbol{q})$ is the maximizer of $\beta U_{f}\left(x_{f}\right)-q_{s(f) f} x_{f}$ which yields $x_{f}^{*}(q)=U_{f}^{\prime-1}\left(q_{s(f) f} / \beta\right)$ the rate value of each $f \in F$ which corresponds to the flow rate control. Finally, $\boldsymbol{p}(\boldsymbol{q})$ is just the stationary distribution of the proposed MAC protocol under suitable values of the backoff timers $R_{l}$. To this end, it suffices to observe that the term $-\sum_{I \in \mathcal{I}} p_{I} \log \left(p_{I}\right)+\sum_{I \in \mathcal{I}} p_{I} \sum_{l \in L i(n)} a_{I l} \alpha_{l} z_{n m}$ (subject to the constraints $\sum_{I \in \mathcal{I}} p_{I}=1, p_{I} \geq 0, I \in \mathcal{I}$ ) is just (10) with $\log \alpha_{l} R_{l}=\alpha_{l} z_{n m}$, i.e., $R_{l}=\frac{\exp \left(\alpha_{l} z_{n m}\right)}{\alpha_{l}}, l=(n, m ; c)$.

2) Iterate Computation: Given $\boldsymbol{p}, \boldsymbol{s}$ and $\boldsymbol{x}$ we compute the new iterates for the Lagrangian multipliers using descent methods. Since $D(q)$ is only piecewise differentiable, we resort to the subgradient method [1] which in our case yields exactly equations (7)-(8).

Convergence of the sub-gradient method depends on the stepsize choice. For a constant step-size $\gamma$, which is here of interest for practical purposes, the algorithm is guaranteed to converge to within a interval (which is proportional to $\gamma$ ) of the optimal value. This shows that the solutions presented in section III are an implementation of the optimum solution of the model formulated in section II.

\section{Conclusions}

In this paper, we have presented a completely distributed cross-layer scheme for cognitive radio wireless networks which jointly optimizes the source flow rate, routing, scheduling and spectrum access. The different algorithms have been derived by studying a distributed solution of a suitable network utility maximization problem which accounts for the presence of primary users. We also briefly discussed how to derive a possible implementation from the proposed ideal scheme which is based on a wireless sensor network for spectrum sensing which has been presented in a companion paper.

\section{REFERENCES}

[1] D. Bertsekas, Nonlinear Programming. Athena Scientific, second ed., 1999.

[2] G. Cheng, W. Liu, Y. Li, W. Cheng, "Joint On-Demand Routing and Spectrum Assignment in Cognitive Radio Networks", in IEEE International Conference on Communications (ICC), June 2007.

[3] L. Ding, T. Melodia, S. Batalama, M. Medley, "ROSA: Distributed Joint Routing and Dynamic Spectrum Allocation in Cognitive Radio Ad Hoc Networks," in Proc. of ACM Intl. Conf. on Modeling, Analysis and Simulation of Wireless and Mobile Systems (MSWiM), Tenerife, Canary Islands, Spain, October 2009.

[4] Z. Han, R. Fan and H. Jiang, "Replacement of Spectrum Sensing in Cognitive Radio", IEEE Transactions on Wireless Communications, vol. 8, no. 6, pp. 2819-2826, June 2009.

[5] Y.T. Hou, Y. Shi and D. Sherali, "Optimal Spectrum Sharing for Multihop Software Defined Radio Networks", em in Proc. of IEEE Infocom 2007, MAy 2007.

[6] F. P. Kelly, "Charging and Rate Control for Elastic Traffic," European Transactions on Telecommunications, vol. 8, no. 1, pp. 33-37, 1997.

[7] F. P. Kelly, A. K. Maulloo and D. K. H. Tan, "Rate Control for Communications Networks: Shadow Prices, Proportional Fairness and Stability," Journal of Operations Research Society, vol. 49, no. 4, pp. 237-252, March 1998.

[8] T. Vamsi Krishna and A. Das, "A survey on MAC protocols in OSA networks", Computer Networks, vol. 53, no. 9, pp. 1377-1394, September 2009.

[9] S. Kunniyur and R. Srikant, "End-to-end congestion control schemes: Utility functions, random losses and ECN marks," IEEE/ACM Transactions on networking, vol. 11, no. 5, pp. 689-702, October 2003.

[10] L. Jiang and J Walrand, "A Distributed CSMA Algorithm for Throughput and Utility Maximization in Wireless Networks," accepted by IEEE/ACM Transactions on Networking.

[11] S. H. Low and D. E. Lapsley, "Optimal flow control, I: Basic algorithm and convergence," IEEE/ACM Transactions on networking, vol. 7, no. 6, pp. 861-874, December 1999.

[12] L. Ma, X. Han, C. Shen, "Dynamic Open Spectrum Sharing MAC protocols for wireless ad hoc networks, in Proc. of IEEE DySPAN 2005, November 2005.

[13] J. Mo and J. Walrand, "Fair end-to-end window based congestion control", in IEEE.ACM Transactions on Networking, Vol .8, No. 5, October 2000.

[14] L. Pescosolido, C. Petrioli, "Designing a Wireless Spectrum Sensor Network to Support Cognitive Radio Operations", submitted for publication.

[15] Sendora Project, http://www.sendora.eu.

[16] M. Thoppian, S. Venkatesan, R. Prakash, R. Chandrasekaran, "MAC layer scheduling in multi-hop wireless networks, in Proc. of the IEEE WoWMoM 2006, June 2006.

[17] L. Tassiulas, "Scheduling and performance limits of networks with constatly varying topology, IEEE Transactions on Information Theory, pp. 1067-1083, May 1997. 